\title{
THE NEED TO IMPLEMENT FRAILTY IN THE INTERNATIONAL CLASSIFICATION OF DISEASE (ICD)
}

\author{
J. MUSCEDERE
}

\begin{abstract}
Department of Critical Care Medicine, Queen's University, Scientific Director, Canadian Frailty Network, Kingston, Ontario, Canada. Corresponding author: Dr. John Muscedere, Kingston Health Sciences Center Watkins C, 76 Stuart Street, Kingston, Ontario K7L 2V3, Phone: 613 549 6666 ext. 4642, Email: John.Muscedere@Kingstonhsc.ca
\end{abstract}

J Frailty Aging 2020;9(1)2-3

Published online January 10, 2020, http:/ / dx.doi.org/10.14283/jfa.2020.2

Aging in humans is highly heterogeneous and its variability increases with increasing age (1). As a consequence, chronological age is of limited utility for diagnosis, prognostication and treatment guidance. In addition, age by itself is of limited use for the assessment of population health, for the evaluation of initiatives designed to promote healthy aging and for health/social care planning. For these purposes, we continue to rely on age augmented by the reporting of disease through the international classification of disease (ICD) (2). However, as societies age around the world, there is a need to improve the ability to evaluate population health including the maintenance of physical and cognitive function from a holistic point of view and this can be done by enhancing ICD reporting.

ICD is a common language for the reporting and monitoring of disease in 100 countries around the world. ICD codes are widely used by clinicians, funders, governments, public health agencies and insurance providers to report diagnoses. This data can be used to track disease prevalence, epidemics and allows for comparisons across ages, geographic areas including the reporting of worldwide, national or regional mortality and morbidity statistics. ICD data can also be used to predict future health care expenditures and guide research and development of new therapies and practices. These quotes from the World Health Organization (WHO) illustrate the importance of ICD codes: "The ICD is the foundation for identification of health trends and statistics globally." and "ICD code analysis is an essential component of research and development" (2).

Since age has not been traditionally viewed as a disease, it is poorly represented in the ICD disease classification system. As examples, in ICD - 10 which is the currently used version of ICD, there is only a code for age-related physical debility (R54) (3). In the newly released version, ICD-11, old age is classified under general symptoms (code MG2A) and there is an extension code for age related disease (XT9T) (4). There is no ability to report on overall physical and cognitive function and the cumulative impact of disease, lifestyles and socioeconomic factors on the individual which is crucial with the aging of populations worldwide.

A way to move forward from the purely disease-based model of reporting to one which incorporates both disease and holistic elements is to develop the reporting of frailty in the ICD classification system. Frailty is a concept that incorporates function and vulnerability and is more predictive of adverse outcomes including mortality, response to treatment, need for institutionalization and increased utilization of healthcare resources than chronological age alone (5). The prevalence of frailty increases with age but aging is not a surrogate for frailty and frailty prevalence varies based on both socioeconomic and health determinants.

The assessment and reporting of frailty through an ICD code would have the following beneficial effects. First of all, frailty assessment should lead to further evaluation, addressment and possible amelioration of the underlying factors contributing to frailty. Further, the use of a frailty ICD code would facilitate capture of clinical and administrative health data thereby leading to the ability to evaluate the delivery of best practices for frail individuals. Frailty ICD data could also incentivize or provide needed information to funders/decision-makers to allocate both health and social care resources based on need. A frailty ICD code may also improve the increasing amount of frailty research since the standardization of frailty characterization in studies needs to be improved. ICD codes are required for the registration of all new drugs and therapies and there is increasing interest in developing therapies specifically targeted at frail individuals. In this regard, drug registration studies are increasingly enrolling aged participants and there is a need to study the differential impact in those with frailty. An ICD code would enable agencies such European Medicines Agency/US Food and Drug Administration to mandate frailty measurement in registration trials enrolling older patients.

There are two possible ways to enhance ICD for the recognition of frailty. The first is to develop a new frailty ICD code. The second is to use ICD codes to determine frailty using a deficit model. For the development of a specific frailty ICD code, the major barrier would be to agree on the model for frailty since frailty has been conceptualized as a phenotype, an accumulation of deficits or multi-dimensional construct. These competing models of frailty have resulted in a wealth of research but in some respects have hindered the implementation of frailty in health and social care systems. As an example, the many frailty instruments described in the literature with variable reporting of their validity, reliability and data collection burdens has led to confusion and the nihilistic attitude that we do not know what frailty is. However, there are clinical instruments that have been extensively used, are useful 


\section{THE JOURNAL OF FRAILTY \& AGING}

for risk/outcome prediction and resource utilization. These instruments can be used on an individual, clinical or population basis. For the development of a frailty ICD code we would need to reach consensus on the underlying conceptual model or capitalize on the overlap between models. The assessment instrument would need to be objective, require little time and be valid across health/social systems. Further there is a need to encompass the concept that frailty is not an all or none phenomena but is variable in severity and there would need to be a way for the ICD code to capture this. Lastly, the inertia to implement systematic frailty assessment would need to be overcome but the presence of an ICD code may help surmount this. Finally, the development of the sarcopenia ICD-CM (United States) code could act as a guide for development of frailty ICD code (6).

The other option is to use existing ICD codes to develop automatically applied risk models and frailty assessment based on a deficit model. An example is the Hospital Frailty Risk Score (HFRS) which can be calculated automatically from ICD -10 codes and stratifies patients into low, intermediate and high risk of adverse outcomes after emergency admission to acute care facilities (7). HFRS demonstrates fair to moderate overlap with risk determinations based on Fried and Rockwood frailty scales. The HFRS is currently being used in the National Health Service in the United Kingdom for the assessment of quality of care and for planning of services.
In conclusion, a frailty ICD code is desirable, needed and work towards its development should commence. A frailty ICD code would promote the creation and development of holistic models of care instead of disease-based care leading to better care and perhaps development of new therapies/care models. There are challenges to the development of an ICD frailty code but these can be overcome. A less desirable but possible alternative is to develop frailty models that are widely applicable based on existing ICD codes.

Conflict of interest: Dr. Muscedere is the Scientific Director for the Canadian Frailty Network which is funded by the Government of Canada.

\section{References}

1. Clegg A, Bates C, Young J, et al. Development and validation of an electronic frailty index using routine primary care electronic health record data. Age Ageing 2016;45(3):353-60.

2. International Classification of Diseases (ICD) Information Sheet. 2019 [cited 2019 December 4]; Available from: https://www.who.int/classifications/icd/factsheet/en/.

3. ICD-10 online versions. [cited 2019 December 4]; Available from: https://www.who int/classifications/icd/icdonlineversions/en/.

4. ICD-11. [cited 2019 December 4]; Available from: https://icd.who.int/en

5. Clegg A, Young J, Iliffe S, et al. Frailty in elderly people. Lancet 2013;381(9868):752-62.

6. Vellas B, Fielding RA, Bens C, et al. Implications of ICD-10 for Sarcopenia Clinical Practice and Clinical Trials: Report by the International Conference on Frailty and Sarcopenia Research Task Force. J Frailty Aging 2018;7(1):2-9.

7. Gilbert T, Neuburger J, Kraindler J, et al. Development and validation of a Hospital Frailty Risk Score focusing on older people in acute care settings using electronic hospital records: an observational study. Lancet 2018;391(10132):1775-82. 